\title{
Dietary behaviour of German adults differing in levels of sport activity
}

\author{
Roma Beitz ${ }^{1, *}$, Gert BM Mensink, Yvonne Henschel ${ }^{2}$, Beate Fischer ${ }^{3}$ and \\ Helmut F Erbersdobler ${ }^{2}$ \\ ${ }^{1}$ Robert Koch Institute, Seestrasse 10, D-13353 Berlin, Germany: ${ }^{2}$ Christian-Albrechts-University, Kiel, Germany: \\ ${ }^{3}$ GSF - National Research Centre for Environment and Health, Institute of Epidemiology, Neuherberg, Germany
}

Submitted 10 February 2003: Accepted 12 June 2003

\begin{abstract}
Objective: To analyse the dietary behaviour of persons engaging in different levels of sport activity.

Design: A cross-sectional survey analysis.

Setting: A population-based sample of German adults.

Subjects: A total of 1756 men and 2254 women participating in the German National Health Interview and Examination Survey and the integrated German Nutrition Survey 1998.

Results: Among both genders, the median micronutrient intakes of active persons were more preferable than those of sedentary persons when German current reference values were used as a criterion. The median nutrient densities were also higher in the diet of active persons, especially those of vitamin E, calcium, magnesium, and among women also those of folate and vitamin C. On average, active persons consumed higher amounts of fruit/vegetable juice, drinking water, milk products (including cheese) and fruits. Active men additionally consumed higher amounts of vegetables and vegetable fat. The contribution of dietary supplements to the total nutrient intake was $3 \%$, on average, for active men and women. Compared with sedentary persons, this contribution was significantly higher for vitamins, and among men also for calcium and magnesium.

Conclusion: There was no indication of an impaired micronutrient intake of active and moderately active persons compared with sedentary persons.
\end{abstract}

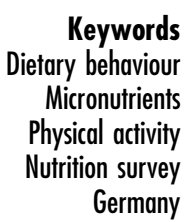

Regular physical activity in leisure time contributes to a healthy lifestyle ${ }^{1-3}$. For most forms of leisure-time activities no special diet is required ${ }^{4}$. However, there are many vitamins and minerals that are involved in physiological processes important to ensure physical performance. Antioxidant vitamins $\mathrm{C}$ and $\mathrm{E}$ are essential for quenching or removal of free radicals. B vitamins act as coenzymes in carbohydrate and amino acid metabolism, in mitochondrial electron transport and in red blood cell synthesis. Magnesium and iron are required as enzyme activators in cellular reactions like the synthesis of ATP and haemoglobin, and in those processes maintaining muscle and nerve excitability. Calcium and potassium participate as electrolytes in, for example, muscle contraction ${ }^{4,5}$.

A high physical activity level is likely to be associated with both increased utilisation of micronutrients and increased nutrient losses via sweat, urine and faeces, which have to be replaced through the diet. Therefore, persons who are physically active could be advised to consume higher amounts of micronutrients than sedentary persons. The aim of the present study was to analyse the micronutrient intakes of persons engaging in different levels of sport activity. The consumption of specified foods as well as demographic characteristics were also examined. The analysis is based on German survey data collected in 1998.

\section{Methods}

A total of 7124 persons participated in the representative German National Health Interview and Examination Survey 1998. The study sample, reflecting the free-living adult population aged 18-79 years, was drawn from population registries, stratified by age, gender, community size and federal state. A sub-sample of 4030 persons participated in the integrated German Nutrition Survey ${ }^{6-8}$. All participants completed a self-administered questionnaire about diet and health-related issues including education, household income, profession, physical activity, smoking habits and vegetarian diet (defined as a 
diet without meat). A physician measured height and weight during a medical examination. All participants underwent a face-to-face drug consumption interview.

The Nutrition Survey participants were interviewed face-to-face about their dietary behaviour of the preceding four weeks. Trained nutritionists asked comprehensively for frequencies, amounts and portion sizes of all foods and beverages consumed using DISHES 98, a validated dietary assessment software based on the dietary history method? In addition, standardised tableware comprising plates, cups, glasses, bowls and spoons, as well as food templates, were used to improve the estimation of portion sizes. Using the German Food Code and Nutrient Data Base, version II.3, quantitative information on foods and beverages was transformed into daily nutrient intakes for each individual ${ }^{10}$.

During the dietary interview the participants also reported their use of vitamin and mineral supplements. The frequencies of intake and brand names of vitamins B, $\mathrm{C}$ and $\mathrm{E}$, folate, multivitamins and mineral supplements were assessed. The units consumed were verified with data from the face-to-face drug consumption interview. Using a new supplement composition database developed by the GSF - National Research Centre for Environment and Health, Institute of Epidemiology and updated by the Robert Koch Institute, information on dietary supplementation was transformed into supplemental nutrient intakes per day. Finally, dietary and supplemental nutrient intakes were summed up to total nutrient intakes per day for each participant ${ }^{11}$.

Information on education, household income and profession was used to create an index of socio-economic status (SES), which was grouped into a low, middle and upper category. Body mass index (BMI) was calculated as weight (in kilograms) divided by the square of height (in metres). Smoking habits were classified as never smoking, ex-smoking and current smoking. Following a predominant (almost always) or exclusive meatless diet was characterised as vegetarianism. Persons with a reported dietary supplementation frequency of at least once a week were defined as regular users, whereas persons who supplemented their diet less frequently were defined as irregular users. Persons who did not use supplements were defined as non-users. Using the question 'How often do you engage in sports?' with five possible answer categories (no sport activity, <1h/week, 1-2h/week, $2-4 \mathrm{~h} /$ week and $>4 \mathrm{~h} /$ week), persons were assigned into three groups: sedentary persons, persons with a regular sport activity of $<2 \mathrm{~h} /$ week (moderately active) and persons with a regular sport activity of $\geq 2 \mathrm{~h} /$ week (active).

We calculated the percentages of persons in the different sport activity groups according to demographic characteristics. Differences were tested using the Cochran-Mantel-Haenszel test for linear trend. Intakes of vitamins and minerals, micronutrient density and consumption of specified foods of persons in the different activity groups are presented as median and interquartile range. Differences were tested using the Median test within the SAS procedure PROC NPAR1WAY ${ }^{12}$. Micronutrient intake was calculated as the percentage of the current German reference intake ${ }^{13}$. Reaching $100 \%$ of the reference means the person has a nutrient intake equal to the reference level. The reference values were calculated on an individual level depending on age, gender and, among women, on eventual specific living conditions like pregnancy or lactation. Different percentages among the activity groups were tested using the Chi-square test. All statistical analyses were performed for genders separately, using the SAS software package version 8.2 (SAS Institute, Inc., Cary, NC, USA). Differences with a $P$-value of $\leq 0.05$ were considered statistically significant.

\section{Results}

Information on sport activity was available from a total of 1756 men and 2254 women. The proportion of active persons was $24 \%$ for men and $17 \%$ for women. Conducting sports moderately was more prevalent in women (40\%) than in men (33\%) $(P \leq 0.001)$. In Table 1 , persons engaging in different levels of sport activity are described by demographic characteristics.

The percentage of active persons was higher among both men and women with a high SES and also among vegetarians. The percentage of active persons declined, however, with increasing age and BMI. The percentage of active men was lower among current smokers, but higher among regular supplement users.

Micronutrient intakes from foods (without supplements) of persons engaging in different levels of sport activity are presented in Table 2. In general, both active and moderately active persons had higher micronutrient intakes than sedentary persons except for vitamin $\mathrm{B}_{12}$, which was lower. Among men, the intake of almost all micronutrients also differed considerably between active and moderately active persons (on average 10\%). The highest differences were observed between active and sedentary persons with respect to vitamin E, i.e. $22 \%$ for men (13.6 vs. $11.1 \mathrm{mg} \mathrm{day}^{-1}$ ) and $15 \%$ for women (11.3 vs. $9.8 \mathrm{mg} \mathrm{day}^{-1}$ ). Calcium intake was also considerably higher among active compared with sedentary persons. In general, women had lower micronutrient intakes than men.

The higher micronutrient intakes from foods of active and moderately active compared with sedentary persons (Table 2) may partly be attributed to a higher total energy intake. However, the nutrient densities of vitamin $\mathrm{E}$, calcium and magnesium were also higher for both active and moderately active compared with sedentary persons (Table 3). The highest differences were observed for calcium among men (11\%) and for vitamin E among women (12\%). In addition, active women had higher densities of folate and vitamin $\mathrm{C}$ than did sedentary 
Table 1 Percentage of persons engaging in different levels of sport activity according to demographic characteristics

\begin{tabular}{|c|c|c|c|c|c|c|c|c|}
\hline & \multirow[b]{2}{*}{$N$} & \multicolumn{3}{|c|}{ Men } & \multirow[b]{2}{*}{$N$} & \multicolumn{3}{|c|}{ Women } \\
\hline & & $\begin{array}{l}\text { Sedentary } \\
(n=741)\end{array}$ & $\begin{array}{c}\text { Moderately } \\
\text { active } \\
(n=587)\end{array}$ & $\begin{array}{c}\text { Active } \\
(n=428)\end{array}$ & & $\begin{array}{l}\text { Sedentary } \\
(n=983)\end{array}$ & $\begin{array}{l}\text { Moderately } \\
\text { active } \\
(n=894)\end{array}$ & $\begin{array}{c}\text { Active } \\
(n=377)\end{array}$ \\
\hline \multicolumn{9}{|l|}{ Age (years) } \\
\hline $18-24$ & 204 & 20.6 & 34.8 & 44.6 & 253 & 24.5 & 45.9 & 29.6 \\
\hline $25-34$ & 339 & 36.3 & 36.9 & 26.8 & 470 & 36.6 & 45.1 & 18.3 \\
\hline $35-44$ & 381 & 42.0 & 34.9 & 23.1 & 498 & 39.2 & 44.0 & 16.9 \\
\hline $45-54$ & 277 & 40.4 & 36.5 & 23.1 & 379 & 45.1 & 39.8 & 15.0 \\
\hline $55-64$ & 344 & 48.3 & 33.4 & 18.3 & 389 & 52.7 & 32.9 & 14.4 \\
\hline $65-79$ & 211 & 65.4 & 19.9 & $14.7^{\star \star \star}$ & 265 & 67.2 & 25.7 & $7.2^{\star \star \star}$ \\
\hline \multicolumn{9}{|c|}{ Body mass index $\left(\mathrm{kg} \mathrm{m}^{-2}\right)$} \\
\hline$\leq 24.9$ & 570 & 36.0 & 32.8 & 31.2 & 1114 & 34.0 & 43.4 & 22.6 \\
\hline $25.0-29.9$ & 861 & 40.7 & 34.7 & 24.6 & 673 & 47.0 & 39.4 & 13.7 \\
\hline$\geq 30.0$ & 325 & 57.2 & 31.1 & $11.7^{\star \star \star}$ & 467 & 61.7 & 31.3 & $7.1^{\star \star \star}$ \\
\hline \multicolumn{9}{|c|}{ Socio-economic status } \\
\hline Lower & 302 & 53.3 & 24.8 & 21.9 & 523 & 58.1 & 29.1 & 12.8 \\
\hline Middle & 988 & 43.6 & 33.1 & 23.3 & 1270 & 43.0 & 39.8 & 17.2 \\
\hline Upper & 452 & 32.3 & 40.0 & $27.7^{\star \star \star}$ & 446 & 27.8 & 51.8 & $20.4^{\star \star \star}$ \\
\hline \multicolumn{9}{|c|}{ Smoking status } \\
\hline Never & 647 & 34.6 & 33.7 & 31.7 & 1251 & 43.8 & 39.5 & 16.7 \\
\hline Past & 512 & 45.3 & 33.6 & 21.1 & 331 & 38.1 & 42.9 & 19.0 \\
\hline Current & 596 & 47.7 & 33.1 & $19.3^{* * *}$ & 668 & 46.0 & 38.5 & $15.6^{\mathrm{NS}}$ \\
\hline \multicolumn{9}{|c|}{ Vegetarianism } \\
\hline No & 1710 & 42.8 & 33.5 & 23.7 & 2077 & 44.9 & 39.2 & 15.9 \\
\hline Yes & 45 & 22.2 & 28.9 & $48.9^{\star \star \star}$ & 172 & 27.9 & 44.8 & $27.3^{\star \star \star}$ \\
\hline \multicolumn{9}{|c|}{ Supplementation } \\
\hline Never & 1131 & 46.0 & 33.1 & 21.0 & 1262 & 45.6 & 38.7 & 15.7 \\
\hline Irregularly & 316 & 35.1 & 36.1 & 28.8 & 500 & 38.6 & 43.0 & 18.4 \\
\hline Regularly & 309 & 35.6 & 32.0 & $32.4^{\star \star \star}$ & 492 & 43.5 & 38.8 & $17.7^{\mathrm{NS}}$ \\
\hline
\end{tabular}

Trends in the percentages within the activity groups according to demographic characteristics were tested using the Cochran-MantelHaenszel test for linear trend: ${ }^{\star \star \star}, P \leq 0.001 ;{ }^{N S}$, not significant.

Table 2 Micronutrient intakes from foods (without supplements) of persons engaging in different levels of sport activity

\begin{tabular}{|c|c|c|c|c|c|c|c|c|c|c|c|c|}
\hline & \multicolumn{4}{|c|}{ Sedentary } & \multicolumn{4}{|c|}{ Moderately active } & \multicolumn{4}{|c|}{ Active } \\
\hline & Median & $\mathrm{P}_{25}$ & $P_{75}$ & $P^{\mathrm{a}}$ & Median & $\mathrm{P}_{25}$ & $\mathrm{P}_{75}$ & $P^{\mathrm{b}}$ & Median & $\mathrm{P}_{25}$ & $\mathrm{P}_{75}$ & $P^{c}$ \\
\hline MEN & \multicolumn{4}{|c|}{$(n=741)$} & \multicolumn{4}{|c|}{$(n=587)$} & \multicolumn{4}{|c|}{$(n=428)$} \\
\hline Vitamin $\mathrm{B}_{1}\left(\mathrm{mg}\right.$ day $\left.^{-1}\right)$ & 1.48 & 1.20 & 1.85 & NS & 1.48 & 1.22 & 1.84 & 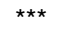 & 1.61 & 1.30 & 1.98 & ** \\
\hline Vitamin $\mathrm{B}_{2}\left(\mathrm{mg} \mathrm{day}^{-1^{\prime}}\right)$ & 1.80 & 1.49 & 2.23 & NS & 1.83 & 1.45 & 2.30 & $\star \star \star *$ & 2.01 & 1.64 & 2.47 & 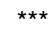 \\
\hline Vitamin $\mathrm{B}_{6}\left(\mathrm{mg} \mathrm{day}^{-1}\right)$ & 2.06 & 1.71 & 2.50 & NS & 2.10 & 1.75 & 2.51 & 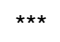 & 2.25 & 1.89 & 2.65 & 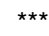 \\
\hline Folate equivalents $\left(\mu \mathrm{g}\right.$ day $^{-1}$ ) & 262.07 & 212.76 & 318.49 & $* *$ & 276.89 & 229.03 & 329.80 & $* * *$ & 300.52 & 242.13 & 372.78 & $* * *$ \\
\hline Vitamin $B_{12}\left(\mu \mathrm{g} \mathrm{day}^{-1}\right)$ & 7.44 & 5.66 & 10.01 & $* *$ & 6.85 & 4.99 & 9.19 & NS & 7.04 & 5.40 & 9.63 & NS \\
\hline Vitamin $C\left(\right.$ mg day $\left.^{-1}\right)$ & 123.43 & 88.80 & 172.24 & $\star *$ & 136.87 & 98.35 & 185.05 & * & 150.09 & 107.37 & 205.25 & $* \star *$ \\
\hline Vitamin $\mathrm{E}\left(\mathrm{mg}\right.$ day $\left.^{-1}\right)$ & 11.10 & 8.45 & 14.33 & $\star *$ & 11.97 & 9.35 & 15.16 & $\star \star \star *$ & 13.55 & 10.53 & 17.57 & 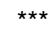 \\
\hline Calcium (g day $\left.{ }^{-1}\right)$ & 1.07 & 0.81 & 1.39 & $* * *$ & 1.16 & 0.90 & 1.48 & $* * *$ & 1.34 & 1.00 & 1.67 & $* * *$ \\
\hline Magnesium ( g day $^{-1}$ ) & 0.46 & 0.37 & 0.54 & $\star \star \star *$ & 0.49 & 0.40 & 0.57 & $\star \star \star *$ & 0.53 & 0.44 & 0.62 & 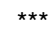 \\
\hline Potassium ( $\left.\mathrm{g} \mathrm{day}^{-1}\right)^{\prime}$ & 3.65 & 3.04 & 4.46 & * & 3.78 & 3.16 & 4.48 & $* \star *$ & 4.14 & 3.40 & 4.86 & $\star * \star *$ \\
\hline Iron $\left(\mathrm{mg} \mathrm{day}^{-1}\right)$ & 15.50 & 12.71 & 19.22 & NS & 15.65 & 13.35 & 19.11 & 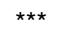 & 17.53 & 14.67 & 21.04 & $\star \star \star *$ \\
\hline WOMEN & \multicolumn{4}{|c|}{$(n=983)$} & \multicolumn{4}{|c|}{$(n=894)$} & \multicolumn{4}{|c|}{$(n=377)$} \\
\hline Vitamin $\mathrm{B}_{1}\left(\mathrm{mg} \mathrm{day}^{-1}\right)$ & 1.10 & 0.92 & 1.32 & ** & 1.15 & 0.95 & 1.37 & NS & 1.12 & 0.92 & 1.37 & NS \\
\hline Vitamin $\mathrm{B}_{2}\left(\mathrm{mg} \mathrm{day}^{-1}\right)$ & 1.41 & 1.15 & 1.71 & * & 1.48 & 1.25 & 1.80 & NS & 1.50 & 1.23 & 1.81 & * \\
\hline Vitamin $\mathrm{B}_{6}\left(\mathrm{mg} \mathrm{day}^{-1}\right)^{\prime}$ & 1.59 & 1.32 & 1.87 & $* *$ & 1.65 & 1.40 & 1.96 & NS & 1.65 & 1.37 & 2.00 & * \\
\hline Folate equivalents ( $\mu \mathrm{g} \mathrm{day}^{-1}$ ) & 221.60 & 181.60 & 268.00 & $* * *$ & 236.67 & 196.07 & 286.02 & NS & 245.19 & 200.05 & 297.40 & $* * *$ \\
\hline Vitamin $B_{12}\left(\mu \mathrm{g} \mathrm{day}^{-1}\right)$ & 4.74 & 3.51 & 6.47 & NS & 4.80 & 3.60 & 6.41 & $\star *$ & 4.33 & 3.15 & 6.04 & * \\
\hline Vitamin C (mg day $\left.{ }^{-1}\right)^{\prime}$ & 127.21 & 92.27 & 173.82 & $* * *$ & 141.58 & 104.63 & 185.84 & NS & 141.26 & 101.80 & 207.90 & * \\
\hline Vitamin $\mathrm{E}\left(\mathrm{mg} \mathrm{day}^{-1}\right)^{\prime}$ & 9.77 & 7.72 & 12.44 & 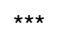 & 10.64 & 8.16 & 13.40 & NS & 11.26 & 8.46 & 13.85 & 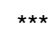 \\
\hline Calcium ( g day $\left.^{-1}\right)$ & 1.00 & 0.78 & 1.24 & $\star \star * \star$ & 1.10 & 0.88 & 1.37 & NS & 1.14 & 0.89 & 1.41 & 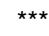 \\
\hline Magnesium ( g day $^{-1}$ ) & 0.38 & 0.31 & 0.45 & $* \star *$ & 0.41 & 0.34 & 0.49 & * & 0.42 & 0.34 & 0.51 & 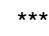 \\
\hline Potassium (g day $\left.{ }^{-1}\right)^{\prime}$ & 3.06 & 2.53 & 3.62 & $* * *$ & 3.22 & 2.70 & 3.79 & NS & 3.28 & 2.75 & 3.99 & ** \\
\hline Iron (mg day ${ }^{-1}$ ) & 12.64 & 10.49 & 14.96 & 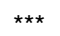 & 13.28 & 11.34 & 15.74 & NS & 13.48 & 11.23 & 16.27 & 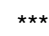 \\
\hline
\end{tabular}

$P_{25}-25$ th percentile; $P_{75}-75$ th percentile.

Differences in dietary intake between a sedentary and moderately active persons, ${ }^{\mathrm{b}}$ moderately active and active persons and ${ }^{\mathrm{c}}$ sedentary and active persons were tested using the Median test: ${ }^{*}, P \leq 0.05 ;{ }^{* \star}, P \leq 0.01 ;{ }^{\star \star \star}, P \leq 0.001 ;{ }^{N S}$, not significant. 
Table 3 Micronutrient density (without supplement intake) of persons engaging in different levels of sport activity

\begin{tabular}{|c|c|c|c|c|c|c|c|c|c|c|c|c|}
\hline & \multicolumn{4}{|c|}{ Sedentary } & \multicolumn{4}{|c|}{ Moderately active } & \multicolumn{4}{|c|}{ Active } \\
\hline & Median & $\mathrm{P}_{25}$ & $\mathrm{P}_{75}$ & $P^{\mathrm{a}}$ & Median & $\mathrm{P}_{25}$ & $\mathrm{P}_{75}$ & $P^{\mathrm{b}}$ & Median & $\mathrm{P}_{25}$ & $\mathrm{P}_{75}$ & $P^{\mathrm{c}}$ \\
\hline MEN & \multicolumn{4}{|c|}{$(n=741)$} & \multicolumn{4}{|c|}{$(n=587)$} & \multicolumn{4}{|c|}{$(n=428)$} \\
\hline Vitamin $\mathrm{B}_{1}\left(\mathrm{mqMJ}^{-1} \mathrm{dav}^{-1}\right)$ & 0.15 & 0.13 & 0.17 & NS & 0.15 & 0.13 & 0.17 & NS & 0.14 & 0.13 & 0.16 & ** \\
\hline Vitamin $\mathrm{B}_{2}\left(\mathrm{mg} \mathrm{MJ}^{-1}\right.$ day $\left.^{-1}\right)$ & 0.18 & 0.16 & 0.20 & NS & 0.18 & 0.16 & 0.21 & NS & 0.18 & 0.16 & 0.20 & NS \\
\hline Vitamin $B_{6}\left(\mathrm{mg} \mathrm{MJ}^{-1}\right.$ day $\left.^{-1}\right)$ & 0.21 & 0.18 & 0.23 & NS & 0.20 & 0.18 & 0.23 & NS & 0.20 & 0.18 & 0.23 & NS \\
\hline Folate equivalents ( $\mu \mathrm{g} \mathrm{MJ}^{-1}$ day $^{-1}$ ) & 25.64 & 22.19 & 29.88 & * & 26.70 & 22.61 & 31.45 & NS & 26.52 & 22.67 & 31.62 & NS \\
\hline Vitamin $\mathrm{B}_{12}\left(\mu \mathrm{g} \mathrm{MJ}^{-1} \mathrm{day}^{-1}\right)$ & 0.72 & 0.58 & 0.92 & $\star \star \star *$ & 0.65 & 0.52 & 0.80 & NS & 0.62 & 0.49 & 0.78 & *** \\
\hline Vitamin $\mathrm{C}\left(\mathrm{mg} \mathrm{MJ}^{-1} \mathrm{day}^{-1}\right)^{\prime}$ & 12.26 & 9.03 & 16.97 & NS & 12.89 & 9.68 & 18.37 & NS & 13.10 & 9.59 & 18.82 & NS \\
\hline Vitamin $\mathrm{E}\left(\mathrm{mg} \mathrm{MJ}^{-1} \mathrm{day}^{-1}\right)$ & 1.08 & 0.90 & 1.30 & ** & 1.14 & 0.93 & 1.42 & * & 1.20 & 0.99 & 1.44 & *** \\
\hline Calcium $\left(\mathrm{g} \mathrm{MJ}^{-1}\right.$ day $\left.^{-1}\right)$ & 0.10 & 0.08 & 0.13 & $\star \star \star *$ & 0.11 & 0.09 & 0.14 & NS & 0.12 & 0.09 & 0.15 & *** \\
\hline Magnesium $\left(\mathrm{g} \mathrm{MJ}^{-1}\right.$ day $\left.^{-1}\right)$ & 0.04 & 0.04 & 0.05 & $* *$ & 0.05 & 0.04 & 0.05 & NS & 0.05 & 0.04 & 0.06 & * \\
\hline Potassium $\left(\mathrm{g} \mathrm{MJ}^{-1} \text { day }^{-1}\right)^{\prime}$ & 0.36 & 0.32 & 0.41 & NS & 0.37 & 0.32 & 0.42 & NS & 0.37 & 0.32 & 0.42 & NS \\
\hline Iron $\left(\mathrm{mg} \mathrm{MJ}^{-1}\right.$ day $\left.^{-1}\right)$ & 1.55 & 1.36 & 1.74 & NS & 1.54 & 1.35 & 1.75 & NS & 1.55 & 1.37 & 1.74 & NS \\
\hline WOMEN & \multicolumn{4}{|c|}{$(n=9 \varepsilon$} & \multicolumn{4}{|c|}{$(n=894)$} & \multicolumn{4}{|c|}{$(n=377)$} \\
\hline Vitamin $\mathrm{B}_{1}\left(\mathrm{mg} \mathrm{MJ}^{-1}\right.$ day $\left.^{-1}\right)$ & 0.15 & 0.13 & 0.17 & * & 0.14 & 0.13 & 0.16 & NS & 0.14 & 0.13 & 0.17 & NS \\
\hline Vitamin $B_{2}\left(\mathrm{mg} \mathrm{MJ}^{-1} \mathrm{day}^{-1}\right)$ & 0.19 & 0.16 & 0.22 & NS & 0.19 & 0.17 & 0.21 & NS & 0.18 & 0.17 & 0.22 & NS \\
\hline Vitamin $B_{6}\left(\mathrm{mg} \mathrm{MJ}^{-1}\right.$ day $\left.^{-1}\right)$ & 0.21 & 0.18 & 0.24 & NS & 0.21 & 0.18 & 0.23 & NS & 0.21 & 0.18 & 0.24 & NS \\
\hline Folate equivalents $\left(\mu \mathrm{g} \mathrm{MJ}^{-1}\right.$ day $\left.^{-1}\right)$ & 29.03 & 24.53 & 34.16 & NS & 29.56 & 25.29 & 35.40 & * & 30.92 & 26.74 & 36.64 & *** \\
\hline Vitamin $\mathrm{B}_{12}\left(\mu \mathrm{gMJ} \mathrm{J}^{-1} \mathrm{dav}^{-1}\right)$ & 0.62 & 0.50 & 0.81 & * & 0.59 & 0.48 & 0.78 & ** & 0.54 & 0.42 & 0.72 & *** \\
\hline Vitamin $\mathrm{C}\left(\mathrm{mg} \mathrm{MJ}^{-1}\right.$ day $\left.^{-1}\right)$ & 16.86 & 11.86 & 23.11 & NS & 17.61 & 12.88 & 24.11 & NS & 18.13 & 13.68 & 24.78 & * \\
\hline Vitamin $\mathrm{E}\left(\mathrm{mg} \mathrm{MJ}^{-1}\right.$ day $\left.^{-1}\right)$ & 1.26 & 1.06 & 1.55 & * & 1.31 & 1.11 & 1.57 & ** & 1.41 & 1.13 & 1.65 & *** \\
\hline Calcium $\left(\mathrm{g} \mathrm{MJ}^{-1}\right.$ day $\left.^{-1}\right)$ & 0.13 & 0.10 & 0.16 & * & 0.14 & 0.11 & 0.17 & NS & 0.14 & 0.12 & 0.18 & ** \\
\hline Magnesium $\left(\mathrm{g} \mathrm{MJ}^{-1}\right.$ day $\left.^{-1}\right)$ & 0.05 & 0.04 & 0.06 & ** & 0.05 & 0.04 & 0.06 & NS & 0.05 & 0.05 & 0.06 & *** \\
\hline Potassium $\left(\mathrm{g} \mathrm{MJ}^{-1} \text { day }^{-1}\right)^{\prime}$ & 0.41 & 0.35 & 0.46 & NS & 0.40 & 0.36 & 0.46 & * & 0.42 & 0.37 & 0.48 & NS \\
\hline Iron $\left(\mathrm{mg} \mathrm{MJ}^{-1}\right.$ day $\left.^{-1}\right)$ & 1.66 & 1.49 & 1.87 & NS & 1.67 & 1.50 & 1.88 & NS & 1.68 & 1.53 & 1.90 & NS \\
\hline
\end{tabular}

$P_{25}-25$ th percentile; $P_{75}-75$ th percentile.

Differences in micronutrient density between a sedentary and moderately active persons, ${ }^{b}$ moderately active and active persons and ${ }^{\mathrm{c}}$ sedentary and active persons were tested using the Median test: ${ }^{\star}, P \leq 0.05 ;{ }^{\star \star}, P \leq 0.01 ;{ }^{* \star}, P \leq 0.001 ;{ }^{N S}$, not significant.

women. On the other hand, the nutrient density of vitamin $\mathrm{B}_{12}$ was higher among sedentary persons compared with both active and moderately active persons. In general, women exceeded men in the density of several nutrients, which was especially obvious for vitamin C, calcium and potassium.

Total micronutrient intakes (including supplements) of persons engaging in different levels of sport activity are shown in Table 4. In general, active as well as moderately active persons had higher total micronutrient intakes than did sedentary persons except for vitamin $\mathrm{B}_{12}$, which was lower. Whereas a higher total intake of several micronutrients was also observed for active compared with moderately active men, for women this was true for vitamin $\mathrm{E}$ and magnesium only. For men, the contribution of supplements to the total micronutrient intake increased with higher engagement in sports: up to 5\% for vitamins and $1 \%$ for minerals, on average. Among women, supplemental mineral intakes were highest for active women (on average $2 \%$ ), and supplemental vitamin intakes were highest for moderately active women (on average 5\%). Both genders supplemented their diet especially with vitamin C, vitamin E, calcium and magnesium. Women had generally lower total nutrient intakes than did men, but higher contributions of supplements to the total intakes, particularly for minerals.

Table 5 compares the total micronutrient intakes of persons engaging in different levels of sport activity with current German reference intakes. Regardless of sport activity level, the percentage of persons with an intake less than $75 \%$ of reference was generally small. Nevertheless, a relatively high percentage of persons consumed less than $75 \%$ of the recommended intake for folate, vitamin $\mathrm{E}$ and calcium. This was especially the case among sedentary persons. For them, the percentage with a low intake of folate ( $66 \%$ of men, $82 \%$ of women), vitamin E (about $35 \%$ ) and calcium (about 20\%) was significantly higher compared with both moderately active and active persons. In general, a relatively high percentage of women had an iron intake less than $75 \%$ of reference.

Whereas the micronutrient intakes of most people ranged between 75 and $150 \%$ of reference, a high percentage of persons - especially men - had micronutrient intakes of more than $150 \%$ of reference. Except for folate, vitamin $\mathrm{B}_{12}$ and iron (women), this percentage was significantly higher among active compared with sedentary persons, and among men also compared with moderately active persons (except for folate and potassium). Concerning vitamin $\mathrm{B}_{12}$, a higher percentage of moderately active, and among men also of sedentary persons, was observed to consume more than $150 \%$ of the reference intake compared with active persons. The highest number of active men exceeding 150\% of reference was observed for potassium (86\%), iron (70\%), vitamin $\mathrm{B}_{6}(57 \%)$ and vitamin $\mathrm{C}(55 \%)$. The highest percentage of active women exceeding $150 \%$ of reference was $62 \%$ for potassium, $52 \%$ for vitamin C, $44 \%$ for vitamin $\mathrm{B}_{6}$ and $40 \%$ for magnesium. 
Table 4 Total micronutrient intakes (including supplements) of persons engaging in different levels of sport activity

\begin{tabular}{|c|c|c|c|c|c|c|c|c|c|c|c|c|}
\hline & \multicolumn{4}{|c|}{ Sedentary } & \multicolumn{4}{|c|}{ Moderately active } & \multicolumn{4}{|c|}{ Active } \\
\hline & Median & $P_{25}$ & $P_{75}$ & $P^{a}$ & Median & $P_{25}$ & $P_{75}$ & $P^{\mathrm{b}}$ & Median & $P_{25}$ & $\mathrm{P}_{75}$ & $P^{\mathrm{c}}$ \\
\hline MEN & \multicolumn{4}{|c|}{$(n=741)$} & \multicolumn{4}{|c|}{$(n=587)$} & \multicolumn{4}{|c|}{$(n=428)$} \\
\hline Vitamin $B_{1}\left(m g\right.$ day $\left.^{-1}\right)$ & 1.55 & 1.22 & 1.94 & NS & 1.54 & 1.27 & 1.97 & $* \star *$ & 1.69 & 1.36 & 2.12 & $\star \star \star$ \\
\hline Vitamin $B_{2}\left(m g\right.$ day $\left.^{-1}\right)$ & 1.83 & 1.51 & 2.35 & NS & 1.91 & 1.53 & 2.44 & *** & 2.09 & 1.71 & 2.74 & $\star \star \star *$ \\
\hline Vitamin $\mathrm{B}_{6}\left(\mathrm{mg}\right.$ day $\left.^{-1}\right)$ & 2.11 & 1.74 & 2.59 & NS & 2.17 & 1.80 & 2.67 & $\star \star *$ & 2.33 & 1.96 & 2.92 & *** \\
\hline Folate equivalents ( $\mu \mathrm{g}$ day $\left.^{-1}\right)$ & 265.27 & 216.51 & 325.53 & $\star \star \star *$ & 285.85 & 237.71 & 343.12 & $* * *$ & 311.93 & 256.99 & 399.32 & $\star \star \star *$ \\
\hline Vitamin $B_{12}\left(\mu \mathrm{g}_{\text {day }}{ }^{-1}\right)$ & 7.59 & 5.71 & 10.17 & $\star \star \star$ & 6.98 & 5.16 & 9.43 & * & 7.40 & 5.58 & 10.06 & NS \\
\hline Vitamin C (mg day $\left.{ }^{-1}\right)$ & 129.39 & 91.67 & 179.38 & $\star \star \star$ & 143.50 & 104.01 & 196.81 & * & 157.71 & 115.56 & 225.63 & $\star \star \star *$ \\
\hline Vitamin $\mathrm{E}\left(\mathrm{mg}\right.$ day $\left.^{-1}\right)$ & 11.48 & 8.65 & 14.84 & $\star \star \star$ & 12.63 & 9.59 & 16.38 & $\star * *$ & 14.96 & 10.88 & 19.22 & $\star \star * *$ \\
\hline Calcium $\left(\mathrm{g}\right.$ day $\left.^{-1}\right)$ & 1.07 & 0.81 & 1.40 & $\star \star \star$ & 1.17 & 0.90 & 1.50 & $* \star *$ & 1.35 & 1.01 & 1.69 & $\star \star \star *$ \\
\hline Magnesium $\left(\mathrm{g}\right.$ day $^{-1}$ ) & 0.45 & 0.38 & 0.56 & $\star \star \star$ & 0.49 & 0.40 & 0.57 & $* \star *$ & 0.54 & 0.45 & 0.64 & $\star \star \star$ \\
\hline Potassium $\left(\mathrm{g} \mathrm{day}^{-1}\right)^{\prime}$ & 3.65 & 3.04 & 4.46 & * & 3.78 & 3.16 & 4.48 & $* * *$ & 4.14 & 3.40 & 4.86 & $\star \star \star *$ \\
\hline Iron $\left(\mathrm{mg}\right.$ day $\left.^{-1}\right)$ & 15.51 & 12.77 & 19.24 & NS & 15.74 & 13.35 & 19.16 & $* \star *$ & 17.65 & 14.68 & 21.13 & $\star \star \star$ \\
\hline WOMEN & \multicolumn{4}{|c|}{$(n=983)$} & \multicolumn{4}{|c|}{$(n=894)$} & \multicolumn{4}{|c|}{$(n=377)$} \\
\hline Vitamin $B_{1}\left(m g\right.$ day $\left.^{-1}\right)$ & 1.15 & 0.94 & 1.44 & $\star \star *$ & 1.21 & 0.99 & 1.48 & NS & 1.19 & 0.95 & 1.51 & NS \\
\hline Vitamin $B_{2}\left(m g\right.$ day $\left.^{-1}\right)$ & 1.47 & 1.18 & 1.84 & $\star *$ & 1.56 & 1.28 & 1.98 & NS & 1.55 & 1.27 & 1.99 & * \\
\hline Vitamin $\mathrm{B}_{6}\left(\mathrm{mg}\right.$ day $\left.^{-1}\right)$ & 1.63 & 1.35 & 1.98 & $\star \star \star$ & 1.74 & 1.44 & 2.15 & NS & 1.71 & 1.41 & 2.16 & ** \\
\hline Folate equivalents ( $\mu \mathrm{g}$ day $^{-1}$ ) & 227.51 & 186.42 & 280.74 & $\star \star \star$ & 247.56 & 200.72 & 306.56 & NS & 252.90 & 206.29 & 327.72 & $\star \star \star$ \\
\hline Vitamin $\mathrm{B}_{12}\left(\mu \mathrm{g}_{\text {day }}{ }^{-1}\right)$ & 4.90 & 3.59 & 6.93 & NS & 4.92 & 3.80 & 6.72 & $* *$ & 4.52 & 3.28 & 6.32 & * \\
\hline Vitamin C (mg day $\left.{ }^{-1}\right)$ & 134.65 & 95.14 & 188.34 & $\star \star \star *$ & 151.05 & 109.43 & 208.06 & NS & 152.41 & 106.54 & 227.95 & $\star *$ \\
\hline Vitamin $\mathrm{E}\left(\mathrm{mg}\right.$ day $\left.^{-1}\right)$ & 10.28 & 8.01 & 13.57 & $\star \star \star$ & 11.32 & 8.59 & 14.67 & * & 11.86 & 8.90 & 15.45 & $\star \star \star$ \\
\hline Calcium $\left(\right.$ g day $\left.^{-1}\right)$ & 1.02 & 0.80 & 1.29 & $\star \star \star$ & 1.12 & 0.89 & 1.40 & NS & 1.16 & 0.89 & 1.44 & $\star \star \star$ \\
\hline Magnesium (g day $\left.{ }^{-1}\right)$ & 0.38 & 0.31 & 0.46 & $\star \star \star$ & 0.41 & 0.34 & 0.50 & * & 0.43 & 0.35 & 0.52 & $\star \star \star$ \\
\hline Potassium $\left(\mathrm{g} \mathrm{day}^{-1}\right)^{\prime}$ & 3.06 & 2.53 & 3.62 & $\star \star \star ~$ & 3.22 & 2.71 & 3.79 & NS & 3.28 & 2.75 & 3.99 & $\star \star *$ \\
\hline Iron $\left(\mathrm{mg} \mathrm{day}^{-1}\right)$ & 12.73 & 10.62 & 15.07 & $\star \star \star$ & 13.49 & 11.39 & 15.91 & NS & 13.75 & 11.26 & 16.54 & $\star \star \star$ \\
\hline
\end{tabular}

$P_{25}-25$ th percentile; $P_{75}-75$ th percentile

Differences in total intake between ${ }^{\mathrm{a}}$ sedentary and moderately active persons, ${ }^{\mathrm{b}}$ moderately active and active persons and ${ }^{\mathrm{c}}$ sedentary and active persons were tested using the Median test: ${ }^{*}, P \leq 0.05 ;{ }^{\star \star}, P \leq 0.01 ;{ }^{\star \star \star}, P \leq 0.001 ;{ }^{N S}$, not significant.

Table 5 Percentage of persons with total micronutrient intake less than $75 \%$ and more than $150 \%$ of the reference value, by level of sport activity

\begin{tabular}{|c|c|c|c|c|c|c|c|c|c|c|c|c|}
\hline & \multicolumn{6}{|c|}{ Less than $75 \%$ of reference } & \multicolumn{6}{|c|}{ More than $150 \%$ of reference } \\
\hline & Sedentary & $P^{\mathrm{a}}$ & $\begin{array}{l}\text { Moderately } \\
\text { active }\end{array}$ & $P^{\mathrm{b}}$ & Active & $P^{\mathrm{c}}$ & Sedentary & $P^{\mathrm{a}}$ & $\begin{array}{l}\text { Moderately } \\
\text { active }\end{array}$ & $P^{\mathrm{b}}$ & Active & $P^{\mathrm{c}}$ \\
\hline MEN & \multicolumn{2}{|c|}{$(n=741)$} & \multicolumn{2}{|l|}{$(n=587)$} & \multicolumn{2}{|c|}{$(n=428)$} & \multicolumn{2}{|c|}{$(n=741)$} & \multicolumn{2}{|l|}{$(n=587)$} & \multicolumn{2}{|c|}{$(n=428)$} \\
\hline Vitamin $\mathrm{B}_{1}\left(\mathrm{mg} \mathrm{day}^{-1}\right)$ & 3.2 & NS & 4.1 & NS & 3.0 & Ns & 37.0 & NS & 36.3 & $\star *$ & 44.6 & * \\
\hline Vitamin $B_{2}\left(\right.$ mg day $\left.^{-1}\right)$ & 1.6 & NS & 2.9 & NS & 1.4 & NS & 39.7 & NS & 40.2 & $\star \star \star *$ & 52.8 & 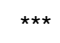 \\
\hline Vitamin $\mathrm{B}_{6}\left(\mathrm{mg} \mathrm{day}^{-1}\right)$ & 1.4 & NS & 1.9 & NS & 0.9 & NS & 43.5 & NS & 45.7 & $\star * \star *$ & 57.0 & *** \\
\hline Folate equivalents ( $\mu \mathrm{g} \mathrm{day}^{-1}$ ) & 66.0 & $\star * *$ & 56.9 & $\star \star \star *$ & 43.9 & 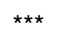 & 2.2 & NS & 2.0 & NS & 4.0 & NS \\
\hline Vitamin $B_{12}\left(\mu \mathrm{g} \mathrm{day}^{-1}\right)$ & 0.8 & NS & 0.7 & NS & 1.4 & NS & 88.7 & $\star *$ & 83.5 & * & 87.6 & NS \\
\hline Vitamin C (mg day $\left.{ }^{-1}\right)$ & 12.2 & NS & 9.9 & NS & 8.6 & NS & 37.5 & $\star \star * *$ & 47.0 & * & 54.9 & *** \\
\hline Vitamin $\mathrm{E}\left(\mathrm{mg} \mathrm{day}^{-1}\right)$ & 36.3 & $\star *$ & 29.5 & $* \star *$ & 19.6 & $\star \star * *$ & 9.2 & NS & 10.7 & 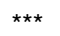 & 19.9 & *** \\
\hline Calcium $\left(\mathrm{g} \mathrm{day}^{-1}\right)$ & 19.7 & 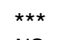 & 12.6 & ** & 7.2 & *** & 20.0 & * & 24.4 & 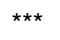 & 34.6 & *** \\
\hline Magnesium (g day ${ }^{-1}$ ) & 3.0 & NS & 2.6 & $\star * *$ & 0.2 & $\star *$ & 31.4 & * & 37.0 & $\star \star \star \star ~$ & 50.7 & *** \\
\hline Potassium ( g day $\left.^{-1}\right)^{\prime}$ & 0.1 & NS & 0.5 & NS & - & NS & 76.1 & * & 81.8 & NS & 86.0 & *** \\
\hline Iron $\left(\mathrm{mg}\right.$ day $\left.^{-1}\right)$ & 0.5 & NS & 0.9 & NS & 0.5 & NS & 53.6 & NS & 57.4 & 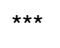 & 70.3 & *** \\
\hline WOMEN & \multicolumn{2}{|c|}{$(n=983)$} & \multicolumn{2}{|l|}{$(n=894)$} & \multicolumn{2}{|c|}{$(n=377)$} & \multicolumn{2}{|c|}{$(n=983)$} & \multicolumn{2}{|l|}{$(n=894)$} & \multicolumn{2}{|c|}{$(n=377)$} \\
\hline Vitamin $\mathrm{B}_{1}\left(\mathrm{mg} \mathrm{day}^{-1}\right)$ & 8.7 & * & 5.9 & NS & 8.0 & NS & 19.9 & NS & 23.3 & NS & 25.7 & * \\
\hline Vitamin $\mathrm{B}_{2}\left(\mathrm{mg} \mathrm{day}^{-1}\right)^{\prime}$ & 6.0 & * & 3.7 & NS & 5.6 & NS & 25.8 & $\star *$ & 32.3 & NS & 33.7 & ** \\
\hline Vitamin $\mathrm{B}_{6}\left(\mathrm{mg}\right.$ day $\left.^{-1}\right)$ & 3.4 & * & 1.7 & NS & 3.2 & NS & 34.0 & *** & 44.6 & NS & 43.8 & *** \\
\hline Folate equivalents ( $\mu \mathrm{g} \mathrm{day}^{-1}$ ) & 81.7 & $\star * *$ & 73.4 & NS & 69.2 & $* \star *$ & 2.5 & NS & 2.4 & NS & 3.7 & NS \\
\hline Vitamin $\mathrm{B}_{12}\left(\mu \mathrm{g}\right.$ day $\left.^{-1}\right)$ & 6.5 & * & 3.9 & $* *$ & 8.2 & NS & 57.0 & NS & 59.4 & $* *$ & 50.4 & * \\
\hline Vitamin $\mathrm{C}\left(\mathrm{mg} \mathrm{day}^{-1}\right)^{\prime}$ & 12.5 & $* * *$ & 7.5 & NS & 10.1 & NS & 40.0 & $* * *$ & 50.0 & NS & 51.5 & *** \\
\hline Vitamin E (mg day $\left.{ }^{-1}\right)$ & 34.5 & 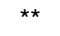 & 28.9 & NS & 25.7 & $\star \star *$ & 11.9 & NS & 14.9 & NS & 17.5 & ** \\
\hline Calcium $\left(\right.$ g day $\left.^{-1}\right)$ & 21.3 & *** & 12.9 & NS & 14.1 & ** & 13.7 & ** & 19.0 & NS & 21.0 & ** \\
\hline Magnesium ( g day $^{-1}$ ) & 4.0 & * & 2.2 & NS & 3.2 & NS & 27.4 & *** & 36.6 & NS & 40.3 & *** \\
\hline Potassium $\left(\mathrm{g} \mathrm{day}^{-1}\right)^{\prime}$ & 1.7 & ** & 0.2 & * & 1.3 & NS & 52.9 & $* * *$ & 61.6 & NS & 61.8 & ** \\
\hline Iron (mg day $\left.{ }^{-1}\right)$ & 17.1 & NS & 16.7 & NS & 18.3 & NS & 11.1 & NS & 11.5 & NS & 10.9 & NS \\
\hline
\end{tabular}

Differences in percentage between a sedentary and moderately active persons, ${ }^{b}$ moderately active and active persons and ${ }^{c}$ sedentary and active persons consuming less than $75 \%$ and more than $150 \%$ of reference were tested using the Chi-square test: ${ }^{*}, P \leq 0.05 ;{ }^{* *}, P \leq 0.01 ;{ }^{* * *}, P \leq 0.001 ;{ }^{N S}$, not significant. 
The consumption of fruit/vegetable juice, drinking water (water as a beverage including tap and mineral water), milk products (including cheese) and fruits, and in addition among men the consumption of vegetables and vegetable fat, was higher for active than for sedentary persons (Table 6). Active persons had a lower consumption of coffee and sausages, and active women also of red meat, than did sedentary persons. This, apart from the higher prevalence of vegetarianism in active persons, may partly explain the generally lower intake of vitamin $\mathrm{B}_{12}$, as red meat and sausages are the main sources of vitamin $B_{12}$ in the diets of the three sport activity groups. Moderately active men also consumed more fruit/vegetable juice, drinking water and milk products (including cheese), but lower amounts of sausages and bread than did sedentary men. Moderately active women consumed more fruit/ vegetable juice, drinking water, milk products (including cheese) and vegetables, but lower amounts of sausages than did sedentary women. Compared with men, women consumed particularly more fruits and drinking water, and less bread, potatoes, red meat, sausages and vegetable fat, regardless of the sport activity level.

\section{Discussion}

The dietary behaviour and demographic characteristics of German adults with different levels of sport activity were examined using data collected in the German National
Health Interview and Examination Survey and the integrated German Nutrition Survey 1998.

Active persons were characterised by lower age and BMI, higher SES and a higher prevalence of vegetarianism. Active men were more likely to be regular supplement users and never smokers. Active persons had more preferable micronutrient intakes than did sedentary persons. This was especially true for folate, for which intake was generally low. Nutrient densities, especially those of vitamin E, calcium and magnesium, and among women also those of folate and vitamin C, were also higher in the diet of active persons. Nevertheless, the micronutrient intakes of sedentary persons were also within recommended ranges in general. Active persons consumed higher amounts of fruit/vegetable juice, drinking water, milk products (including cheese) and fruits, as well as vegetables and vegetable fat (men), than did sedentary persons. Among active persons, dietary supplements contributed to the total micronutrient intake at $3 \%$, on average, which was significantly higher than among sedentary persons regarding vitamins, and for men also calcium and magnesium.

Compared with men, women had generally lower absolute micronutrient intakes because they consumed lower amounts of foods. However, the nutrient density of especially vitamin C, calcium and potassium was higher in the diet of women, probably due to higher consumption of fruits and drinking water. On the other hand, the

Table 6 Consumption of specified foods $\left(\mathrm{g} \mathrm{day}^{-1}\right)$ by persons engaging in different levels of sport activity

\begin{tabular}{|c|c|c|c|c|c|c|c|c|c|c|c|c|}
\hline & \multicolumn{4}{|c|}{ Sedentary } & \multicolumn{4}{|c|}{ Moderately active } & \multicolumn{4}{|c|}{ Active } \\
\hline & Median & $\mathrm{P}_{25}$ & $\mathrm{P}_{75}$ & $P^{a}$ & Median & $\mathrm{P}_{25}$ & $P_{75}$ & $P^{\mathrm{b}}$ & Median & $\mathrm{P}_{25}$ & $P_{75}$ & $P^{\mathrm{c}}$ \\
\hline MEN & \multicolumn{4}{|c|}{$(n=741)$} & \multicolumn{4}{|c|}{$(n=587)$} & \multicolumn{4}{|c|}{$(n=428)$} \\
\hline Milk products (including cheese) & 197.8 & 112.2 & 345.4 & * & 224.9 & 134.0 & 390.8 & * & 283.9 & 160.4 & 480.5 & *** \\
\hline Bread & 176.9 & 126.8 & 229.6 & $\star \star *$ & 162.7 & 120.4 & 212.1 & $\star *$ & 177.7 & 128.3 & 240.2 & NS \\
\hline Vegetables & 129.1 & 87.4 & 192.9 & NS & 139.2 & 98.1 & 206.6 & * & 151.8 & 100.1 & 224.4 & *** \\
\hline Potatoes & 132.6 & 92.1 & 186.9 & NS & 126.6 & 81.2 & 174.9 & NS & 125.3 & 81.6 & 180.4 & NS \\
\hline Fruits & 141.0 & 66.2 & 242.9 & NS & 153.2 & 86.3 & 247.1 & NS & 155.6 & 83.2 & 255.3 & * \\
\hline Red meat & 107.7 & 74.2 & 149.0 & NS & 100.9 & 67.6 & 138.7 & NS & 101.2 & 66.4 & 148.8 & NS \\
\hline Sausages & 60.7 & 36.7 & 91.4 & $\star *$ & 55.0 & 31.8 & 76.4 & NS & 52.0 & 27.7 & 82.5 & ** \\
\hline Vegetable fat & 14.9 & 9.1 & 22.8 & NS & 15.3 & 10.5 & 22.5 & $\star *$ & 17.1 & 11.1 & 26.0 & ** \\
\hline Coffee & 375.0 & 170.0 & 600.0 & NS & 340.0 & 160.7 & 600.0 & * & 300.0 & 48.6 & 514.3 & ** \\
\hline Fruit/vegetable juice & 29.3 & 0.2 & 170.3 & 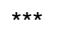 & 73.5 & 0.5 & 215.3 & NS & 97.3 & 0.4 & 302.7 & 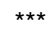 \\
\hline Drinking water & 397.1 & 44.5 & 844.3 & * & 500.0 & 167.1 & 947.0 & * & 584.3 & 172.2 & 1001.2 & *** \\
\hline WOMEN & \multicolumn{4}{|c|}{$(n=983)$} & \multicolumn{4}{|c|}{$(n=894)$} & \multicolumn{4}{|c|}{$(n=377)$} \\
\hline Milk products (including cheese) & 203.9 & 124.5 & 329.2 & $\star \star \star *$ & 234.4 & 153.3 & 366.7 & NS & 251.4 & 153.4 & 387.9 & *** \\
\hline Bread & 125.0 & 91.2 & 161.2 & NS & 122.2 & 90.7 & 161.5 & NS & 114.4 & 82.5 & 157.5 & NS \\
\hline Vegetables & 134.2 & 86.8 & 195.5 & $\star *$ & 146.9 & 103.1 & 209.0 & NS & 141.5 & 92.0 & 214.1 & NS \\
\hline Potatoes & 99.2 & 66.5 & 137.9 & NS & 94.2 & 58.3 & 135.4 & NS & 96.1 & 54.9 & 134.2 & NS \\
\hline Fruits & 171.5 & 103.4 & 280.7 & NS & 182.4 & 111.1 & 290.3 & NS & 197.1 & 126.4 & 302.9 & * \\
\hline Red meat & 71.0 & 47.2 & 103.8 & NS & 70.3 & 46.3 & 96.5 & * & 63.5 & 39.3 & 90.6 & * \\
\hline Sausages & 31.6 & 17.1 & 49.7 & $\star *$ & 28.6 & 14.3 & 47.3 & NS & 24.6 & 8.4 & 39.2 & *** \\
\hline Vegetable fat & 13.1 & 8.0 & 19.1 & NS & 13.0 & 8.3 & 18.6 & NS & 12.5 & 8.3 & 18.1 & NS \\
\hline Coffee & 340.0 & 170.0 & 578.6 & NS & 315.9 & 150.0 & 510.7 & * & 300.0 & 48.6 & 490.0 & ** \\
\hline Fruit/vegetable juice & 48.6 & 0.4 & 171.6 & 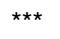 & 86.3 & 2.0 & 244.5 & NS & 94.5 & 1.1 & 266.5 & 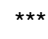 \\
\hline Drinking water & 500.7 & 170.5 & 857.7 & 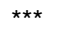 & 641.2 & 300.0 & 1000.0 & NS & 680.6 & 335.7 & 1003.6 & *** \\
\hline
\end{tabular}

$\mathrm{P}_{25}-25$ th percentile; $\mathrm{P}_{75}-75$ th percentile.

Differences in food consumption between ${ }^{\mathrm{a}}$ sedentary and moderately active persons, ${ }^{\mathrm{b}}$ moderately active and active persons and ${ }^{\mathrm{c}}$ sedentary and active persons were tested using the Median test: ${ }^{\star}, P \leq 0.05 ;{ }^{\star \star}, P \leq 0.01 ;{ }^{\star \star \star}, P \leq 0.001 ;{ }^{N S}$, not significant. 
consumption of bread, potatoes, red meat, sausages and vegetable fat was lower than that of men. In contrast to women, micronutrient and food intakes generally increased with increasing level of sport activity in men.

Differences in demographic characteristics between the activity groups have been well documented so far ${ }^{3,14-16}$. Persons who are physically active in leisure-time have less frequently been the subjects of dietary behaviour studies compared with athletes. However, the results presented here are similar to those observed in previous studies. For instance, Mensink and Arab $^{17}$ observed higher intakes of several nutrients, among them $\mathrm{B}$ vitamins, vitamin $\mathrm{E}$, vitamin $C$ and calcium, for physically more active compared with predominantly inactive persons in an elderly and a younger population. Voorrips et $a l^{18}$ reported a trend for physically active elderly women to consume more vegetables, fruits, legumes and nonalcoholic drinks than their sedentary counterparts. Two more studies showed higher intakes of calcium, folate and vitamins $\mathrm{A}, \mathrm{C}$ and $\mathrm{E}$, as well as a higher consumption of fruits and vegetables, but a lower consumption of red and processed meat for more active persons compared with sedentary individuals ${ }^{19,20}$

An additional analysis of mineral status showed no substantial differences between the activity groups (not shown). This is also consistent with previous studies $^{4,17,21,22}$. In general, this may primarily be due to the body's homeostatic control of the status of most minerals. Therefore, a change in mineral intake may not necessarily lead to a different mineral status.

There are several methods used to assess physical activity. The methods include direct observations, activity records, objective mechanical and electronic devices and instruments like accelerometry, and estimation of energy expenditure, e.g. by the doubly labelled water method. These possible assessment instruments are most useful to small-scale studies and for validation purposes. In population surveys a questionnaire-based assessment of physical activity is preferred. This is not as timeconsuming, prohibitive, intrusive and reactive as other methods, and may not lead to alterations of activity behaviour. However, there are several types of questionnaire used in epidemiological research based on either self-reported responses or interviewer administration. We used a simple question allowing for quantitative information on sport activity, but not for type and intensity of the activities. However, since the intention of this study was to compare sedentary persons and more active persons instead of examining exercise or exercise training and total energy expenditure, this question may be appropriate. Nevertheless, standardisation of the content and design of questionnaires used in surveys would be helpful to compare physical activity habits of different populations $^{23,24}$.

The micronutrient intakes were compared with current German reference values ${ }^{13}$. These values have the objective to reflect the nutrient requirements of almost all healthy persons in Germany, on average. However, these requirements vary between individuals. Thus, an intake below the reference intake merely increases the probability of an insufficient intake on a population level. Therefore, we used the level of $75 \%$ of reference to detect micronutrient intakes that are more likely to be suboptimal. Levels above $150 \%$ of reference were those considered to be more than sufficient.

The assessment of dietary intake is generally accompanied by the problem of underreporting of energy intake. According to Goldberg et al. ${ }^{25}$, underreporting is defined as energy intake lower than 1.27 times basal metabolic rate. The percentage of suspected underreporters was higher among sedentary persons, especially for women. However, the present results did not change considerably after the exclusion of suspected underreporters.

The use of dietary supplements was observed to be common among athletes ${ }^{26,27}$. In the present study, a relatively high percentage of supplement users was also seen for persons being active in leisure sports. However, supplemental intake did not contribute substantially to total micronutrient intake, in particular to total mineral intake. This is probably due to the assessment of mineral supplementation. Whereas the nutrition survey participants reported their use of specific vitamin supplements, like vitamins $\mathrm{C}$ and $\mathrm{E}$, and folate supplements, for minerals they were asked for the use of mineral supplements in general. Some participants may not remember all of the mineral supplements used, although open-ended questions for describing type as well as brand name of the supplement taken were used. Therefore, the contribution of mineral supplements to the total mineral intake may be somewhat underestimated.

In conclusion, there is no indication that the micronutrient intakes of active and moderately active persons in Germany are generally insufficient. Since with increasing level of sport activity both utilisation of micronutrients and nutrient losses via sweat, urine and faeces also increase, some active persons may need a higher nutrient intake. In this population most of the active persons meet their requirements. An engagement in sport during leisure time is likely to be a personal choice and, therefore, may be more associated with health consciousness. Persons who have a high physical activity level during their work may not be those who have a high activity level during leisuretime, as has been suggested in a previous publication ${ }^{28}$. The diet of sedentary persons is also generally satisfactory. On a population level, therefore, supplementation with micronutrients is in general not recommended for both persons who are active in leisure-time as well as those with a sedentary lifestyle ${ }^{22,29}$. Because of the beneficial health effects of physical activity in general, a main aim of public health remains the motivation of the population to be engaged in leisure-time physical activity. 


\section{References}

1 Astrup A. Healthy lifestyles in Europe: prevention of obesity and type II diabetes by diet and physical activity. Public Health Nutrition 2001; 4: 499-515.

2 Hardman AE. Physical activity and health: current issues and research needs. International Journal of Epidemiology 2001; 30: $1193-7$.

3 US Department of Health and Human Services (DHHS), Centers for Disease Control and Prevention, National Center for Chronic Disease Prevention and Health Promotion, The President's Council on Physical Fitness and Sports. Physical Activity and Health: A Report of the Surgeon General. Atlanta, GA: DHHS, 1996.

4 Fogelholm GM. Micronutrients: interaction between physical activity, intakes and requirements. Public Health Nutrition 1999; 2: 349-56.

5 Maughan RJ. Role of micronutrients in sport and physical activity. British Medical Bulletin 1999; 55: 683-90.

6 Bellach BM, Knopf H, Thefeld W. Der Bundes-Gesundheitssurvey 1997/98. Gesundheitswesen 1998; 60(Suppl. 2): 59S-68S.

7 Thefeld W, Stolzenberg H, Bellach BM. Bundes-Gesundheitssurvey: Response, Zusammensetzung der Teilnehmer und Non-Responder-Analyse. Gesundheitswesen 1999; 61 (Suppl. 2): 57S-61S.

8 Mensink GBM, Hermann-Kunz E, Thamm M. Der Ernährungssurvey. Gesundheitswesen 1998; 60(Suppl. 2): 83S-6S.

9 Mensink GBM, Haftenberger M, Thamm M. Validity of DISHES 98, a computerised dietary history interview: energy and macronutrient intake. European Journal of Clinical Nutrition 2001; 55: 409-17.

10 Dehne L, Klemm C, Henseler G, Hermann-Kunz E. The German Food Code and Nutrient Data Base (BLS II.2). European Journal of Epidemiology 1999; 15: 355-9.

11 Beitz R, Mensink GBM, Fischer B, Thamm M. Vitamins dietary intake and intake from dietary supplements in Germany. European Journal of Clinical Nutrition 2002; 56: 539-45.

12 SAS Institute, Inc. SAS OnlineDoc ${ }^{\circledR}$, Version 8. Cary, NC: SAS Institute, Inc., 1999.

13 German Nutrition Society/Austrian Nutrition Society/Swiss Society for Nutrition Research/Swiss Nutrition Association. Reference Values for Nutrient Intake. Frankfurt am Main: Umschau/Braus, 2002.

14 Stephens T, Caspersen CJ. The demography of physical activity. In: Bouchard C, Shephard RJ, Stephens T, eds. Physical Activity, Fitness, and Health. International Proceedings and Consensus Statement. Champaign, IL, Human Kinetics Publishers, Inc., 1994; 204-13.

15 Crespo CJ, Keteyian SJ, Heath GW, Sempos CT. Leisure-time physical activity among US adults. Archives of Internal Medicine 1996; 156: 93-8.
16 Pratt M, Macera CA, Blanton C. Levels of physical activity and inactivity in children and adults in the United States: current evidence and research issues. Medicine and Science in Sports and Exercise 1999; 31(Suppl. 11): 526S-33S.

17 Mensink GBM, Arab L. Relationships between nutrient intake, nutritional status and activity levels in an elderly and in a younger population; a comparison of physically more active and more inactive people. Zeitschrift fur Gerontologie 1989; 22: 16-25.

18 Voorrips LE, van Staveren WA, Hautvast JGAJ. Are physically active elderly women in a better nutritional condition than their sedentary peers? European Journal of Clinical Nutrition 1991; 45: 545-52.

19 Mensink GBM, Loose N, Oomen CM. Physical activity and its association with other lifestyle factors. European Journal of Epidemiology 1997; 13: 771-8.

20 Gillman MW, Pinto BM, Tennstedt S, Glanz K, Marcus B, Friedman RH. Relationship of physical activity with dietary behaviours among adults. Preventive Medicine 2001; 32: 295-301.

21 Fogelholm GM, Himberg J-J, Alopaeus K, Gref C-G, Laakso $\mathrm{JT}$, Lehto JJ, et al. Dietary and biochemical indices of nutritional status in male athletes and controls. Journal of the American College of Nutrition 1992; 11: 181-91.

22 Clarkson PM, Thompson HS. Antioxidants: what role do they play in physical activity and health? American Journal of Clinical Nutrition 2000; 72(Suppl. 2): 637S-46S.

23 Ainsworth BE, Montoye HJ, Leon AS. Methods of assessing physical activity during leisure and work. In: Bouchard C, Shephard RJ, Stephens T, eds. Physical Activity, Fitness, and Health. Champaign, IL: Human Kinetics Publishers, Inc., 1994; 146-59.

24 Troiano RP, Macera CA, Ballard-Barbash R. Be physically active each day. How can we know? Journal of Nutrition 2001; 131(Suppl. 2): 451S-60S.

25 Goldberg GR, Black AE, Jebb SA, Cole TJ, Murgatroyd PR, Coward WA, et al. Critical evaluation of energy intake data using fundamental principles of energy physiology: 1. Derivation of cut-off limits to identify under-recording. European Journal of Clinical Nutrition 1991; 45: 569-81.

26 Sobal J, Marquart LF. Vitamin/mineral supplement use among athletes: a review of the literature. International Journal of Sport Nutrition 1994; 4: 320-34.

27 Bazzarre TL, Scarpino A, Sigmon R, Marquart LF, Wu SL, Izurieta M. Vitamin-mineral supplement use and nutritional status of athletes. Journal of the American College of Nutrition 1993; 12: 162-9.

28 Mensink GBM, Deketh M, Mul MDM, Schuit AJ, Hoffmeister H. Physical activity and its association with cardiovascular risk factors and mortality. Epidemiology 1996; 7: 391-7.

29 Lukaski HC. Magnesium, zinc, and chromium nutriture and physical activity. American Journal of Clinical Nutrition 2000; 72(Suppl. 2): 585S-93S. 